\title{
A theoretical analysis of the growth process of an organism and its dependence on various allometric relations
}

\author{
Sudipto Roy ${ }^{1^{*}}$, Priyadarshi Majumdar ${ }^{2}$, Subhankar Ghosh ${ }^{1}$ \\ ${ }^{1}$ Department of Physics, St. Xavier's College, Kolkata, India; \\ *Corresponding Author: roy.sudipto1@gmail.com, sudipto111@yahoo.com \\ ${ }^{2}$ Jyotinagar Bidyasree Niketan Higher Secondary School, Kolkata, India; majumdar_priyadarshi@yahoo.com
}

Received 8 August 2011; revised 8 September 2011; accepted 28 September 2011.

\begin{abstract}
A new mathematical model regarding the growth process of an organism is proposed, based on the role of surplus power (i.e. power intake minus metabolic cost) and having an allometric dependence on mass. Considering its use in growth, a differential equation has been formed, similar to the von Bertalanffy growth function (VBGF). The time dependence of mass and growth rate, obtained from this equation, has been shown graphically to illustrate the roles played by scaling exponents and other parameters. Concepts of optimum mass, saturation mass and the mass corresponding to the highest growth rate have been discussed under the proposed theoretical framework. Information regarding the dependence of effective growth duration on various parameters has been found graphically. The time of occurrence of the highest growth rate and its dependence on various parameters have been explored graphically. A new parameter $(\rho)$ has been defined, which determines the availability of surplus power at different stages of the growth process of an organism. Depending on its value, there can be three distinctly different modes of growth phenomenon, reflected in the change of surplus power with time. The variations of growth and reproduction efficiencies with time and mass have been shown for different values of the scaling exponent. The limitation regarding the practical measurement of growth rate has been discussed using the present model. Some aspects of length-biomass allometry have been explored theoretically and the results have been depicted graphically.
\end{abstract}

Keywords: Allometric Scaling in biology; Von Bertalanffy Growth Function; Biological Growth Model; Growth \& Reproduction Efficiency; Length-Biomass Allometry; Metabolism

\section{INTRODUCTION}

On the basis of experimental evidence as well as theoretical formulations it has already been established that the rates of energy intake and energy loss of a living organism have power-law (like $y=a x^{b}$ ) dependence on the mass of the organism [1-5]. Therefore, one can represent the rate of energy intake $\left(P_{1}\right)$ and rate of energy loss $\left(P_{2}\right)$ by $C_{1} m^{\sigma_{1}}$ and $C_{2} m^{\sigma_{2}}$ respectively; where $C_{1}$ and $C_{2}$ are the constants of proportionality, and $\sigma_{1}$ and $\sigma_{2}$ are the corresponding allometric scaling parameters.

The difference between $P_{1}$ and $P_{2}$ is known as the rate of production of surplus energy $\left(E_{S}=P_{1}-P_{2}\right)$ of the organism which is spent mainly for growth and reproduction processes [6-8]. One can express this surplus energy production rate or surplus power $\left(E_{S}\right)$ as

$$
E_{s}=C_{1} m^{\sigma_{1}}-C_{2} m^{\sigma_{2}} .
$$

Eq.1 can also be derived from the theory of universal phenomenological growth that may be described by a simple law which is expressed as

$$
\mathrm{d} Y(t) / \mathrm{d} t=\alpha(t) Y(t) .
$$

Here, $\alpha(t)$ is a time dependent quantity which represents the specific growth rate of a given variable $Y(t)$. From Eq.2, different types of growth model can be derived. The "Class U1" solution of Eq.2, as described by Castorina et al. [9], gives Gompertz law [10] which is largely applied to describe economical and biological growth phenomena like tumor growth pattern etc [11,12]. Castorina et al. described Eq.1 as the "Class 
U2" solution of Eq.2 [9].

Over the past few decades, several efforts have been made to determine the value of two scaling parameters $\sigma_{1}$ and $\sigma_{2}$. In the study of von Bertalanffy, an assumption of $\sigma_{2}=1$ was specified $[13,14]$. Some studies reveal that the metabolic cost is directly proportional to the mass of an organism, implying $\sigma_{2}=1[4,7]$. Debates are still going on over the value of $\sigma_{1}$. People attempted to explain the value of $\sigma_{1}$ with the help of either metabolic theory of ecology or dynamic energy budget theory. The metabolic theory of ecology is based on the idea that the transport of resources takes place through a fractal-like branching network $[4,15]$. It predicts $\sigma_{1}$ to be $3 / 4$, supported by different experimental observations. The theory of dynamic energy budgets is based on the concept that the rates of basic physiological processes are proportional to body surface area, implying

$\sigma_{1}=2 / 3[16,17]$. A. R. P. Rau offered an explanation for the values of scaling parameters, on the basis of Poiseuille's law of fluid flow [18]. The chemiosmotic theory of energy transduction, combined with the method of quantum statistics, is also applied to explain the variation in scaling exponents [19]. Many such investigations show that $\sigma_{1} \geq 2 / 3$. In some cases, it may be equal to $3 / 4$. Vogel showed that biological processes are controlled by different physical processes like convection, diffusion etc. and the process of mass transport is different for different molecules in an organism [20]. da Silva et al. explained the variation of $\sigma_{1}$ with the help of physical processes like diffusion, convection and anomalous diffusion for different organisms [21,22]. According to the study of Economos [23], the geometry of body surface, which is different for different organisms, is related with energy intake of the organism. da Silva et al. [22] compared the exponent of basal metabolic rates for different organisms and proposed a theoretical explanation for the different values of that exponent. So, growth process can be studied using different values of these scaling exponents.

It has been found through some research [5-7] that, at the initial stage of growth the surplus power $\left(E_{S}\right)$ increases with mass and then it decreases after reaching its peak value at a mass which is known as the optimum mass [7]. Based on this fact, we have shown in our earlier studies that $\sigma_{2}>\sigma_{1}[24,25]$. Thus, the scaling exponent for metabolic cost is found to be greater than the exponent for energy intake. This is an important conclusion which is also found to be valid according to the studies of West et al. [26]. In the present article, we have studied the growth process theoretically, through a model developed by us on the basis of the relation between growth rate and surplus power. Using this mathematical model, an exhaustive analysis of some important aspects of growth mechanism has been made.

\section{MODEL FORMULATION}

In the present study, we have taken $\sigma_{2}=1$, under the consideration that metabolic cost is proportional to the body volume (which is directly proportional to mass), in accordance with some studies [3,7,24,27]. Using this value, Eq.1 is expressed as

$$
E_{s}=C_{1} m^{\sigma_{1}}-C_{2} m .
$$

The above expression of $E_{s}$ has been used in all further calculations in the present article. Since excess energy is mainly used for growth and reproduction, these processes would stop if the surplus power $\left(E_{s}\right)$ ever becomes zero in the life of an organism [7,8]. For a certain value of $m$ (say $M_{h}$ ) we have $E_{s}=0$, as evident from the functional form of $E_{s}$. Using Eq.3, we get

$$
M_{h}=\left(C_{1} / C_{2}\right)^{1 / 1-\sigma_{1}} .
$$

Apart from the processes of growth and reproduction, some excess energy is always required for repair and also to sustain biological processes in situations like sudden environmental fluctuations etc. According to Kozlowski [6] and Sebens [7], some surplus energy $\left(E_{s}\right)$ is always required for a healthy survival of the organism. Therefore, taking $E_{s}>0$, Eqs.3 and 4 yield the following relation

$$
m<\left(C_{1} / C_{2}\right)^{1 / 1-\sigma_{1}} \equiv M_{h} .
$$

Thus, the growth process must stop before reaching the point where $m=M_{h}$. In any growth process, as $t \rightarrow \infty$, mass becomes asymptotic to a certain value (say $M_{a}$ ) where $M_{a}<M_{h}$. The expression of $M_{a}$, the highest attainable mass, has been derived later in this article.

According to some studies [24,25], an organism has a natural tendency to attain the optimum mass $\left(M_{o p t}\right)$ or energetic optimum size (EOS), which corresponds to the greatest surplus power $\left(E_{s}\right)$ [8,12,21]. For $E_{s}$ to be maximum at $m=M_{\text {opt }}$ we must have

1) $\mathrm{d} E_{s} / \mathrm{d} m=0$ and 2) $\mathrm{d}^{2} E_{s} / \mathrm{d} m^{2}<0$ at $m=M_{\text {opt }}$.

The first of the above conditions gives us the following expression of optimum mass $\left(M_{o p t}\right)$

$$
M_{\text {opt }}=\left(C_{1} \sigma_{1} / C_{2}\right)^{1 / 1-\sigma_{1}} .
$$

Applying the second condition we get

$$
\sigma_{1}<1 \text {. }
$$

Substituting $m=M_{o p t}$ in Eq.3 from Eq.6A, the maximum surplus power $\left(E_{s M}\right)$ is obtained as

$$
E_{s M}=C_{1}\left(C_{1} \sigma_{1} / C_{2}\right)^{\sigma_{1} / 1-\sigma_{1}}-C_{2}\left(C_{1} \sigma_{1} / C_{2}\right)^{1 / 1-\sigma_{1}} .
$$


The difference between the rates of energy intake and energetic cost can be termed scope for growth [28] and energy surplus [29] depending on which energetic costs are included. If the costs of building gonad are included then this difference is truly scope for growth. If only the metabolic maintenance costs are included, this difference is an energy surplus, used mainly for growth and reproduction. Experimental observations suggest that the energy allocated for reproduction has an allometric dependence on mass $[7,21,30]$. Therefore, the rate of energy allocation for reproduction $\left(E_{p}\right)$ can be expressed as

$$
E_{p}=C_{3} m^{\sigma_{3}} .
$$

where $\sigma_{3}$ is the allometric scaling exponent and $C_{3}$ is the proportionality constant for the rate of energy spent for reproduction. Both $C_{3}$ and $\sigma_{3}$ are positive quantities. In the present study, we have taken $\sigma_{3}=1$, as proposed by Sebens [7]. Using this value, Eq.8 is written as

$$
E_{p}=C_{3} m
$$

The above expression of $E_{p}$ has been used in all further calculations in this article. The part of the surplus power $\left(E_{s}\right)$ which is not used for reproduction, is mainly used for the growth process. Therefore, the rate of energy allocation for growth $\left(E_{g}\right)$ is given by

$$
E_{g}=E_{s}-E_{p}=C_{1} m^{\sigma_{1}}-\left(C_{2}+C_{3}\right) m
$$

This energy $\left(E_{g}\right)$ causes the mass to increase. Therefore, the rate of variation of mass with time can be expressed as (with proportionality constant scaled to unity)

$$
E_{g}=\mathrm{d} m / \mathrm{d} t=C_{1} m^{\sigma_{1}}-\left(C_{2}+C_{3}\right) m
$$

The above equation is similar in form to the von Bertalanffy growth function (VBGF), which is basically a descriptive mechanistic model derived for fish growth rate, based on a simple mass balance equation [13]. But, instead of two constants of proportionality, we have three constants of proportionality to incorporate separately the effects of energy intake, metabolic cost and reproduction cost in the growth process.

The growth process continues as long as $E_{g}$ remains non-zero. As the organism reaches the state of maximum attainable mass $\left(M_{a}\right), E_{g}$ becomes zero. Therefore, from Eq.11, we obtain

$$
M_{a}=\left(C_{1} / C\right)^{1 / 1-\sigma_{1}},\left[C=C_{2}+C_{3}\right]
$$

with $m=M_{0}$ at $t=0$, the solution to Eq.11 is given by

$$
\begin{aligned}
m(t)= & {\left[C_{1} / C\left(1-\mathrm{e}^{-\beta t}\right)+M_{0}^{1-\sigma_{1}} \mathrm{e}^{-\beta t}\right]^{1 / 1-\sigma_{1}} } \\
& {\left[\beta=C\left(1-\sigma_{1}\right)\right] }
\end{aligned}
$$

Using Eqs.12 and 13 can be expressed as

$$
m(t)=\left[\left(M_{a}\right)^{1-\sigma_{1}}\left(1-\mathrm{e}^{-\beta t}\right)+M_{0}^{1-\sigma_{1}} \mathrm{e}^{-\beta t}\right]^{1 / 1-\sigma_{1}} .
$$

From Eq.14 it is found that, as $t \rightarrow \infty, m \rightarrow M_{a}$. It means that, after a sufficiently long time, the mass becomes almost equal to $M_{a}$. Practically, the organism does not appear to grow in size when its mass is very close to $M_{a}$.

Now using Eqs.11 and 13 one may write the growth rate as

$$
\begin{aligned}
& \mathrm{d} m / \mathrm{d} t=E_{g} \\
& =\left[C_{1} / C\left(1-\mathrm{e}^{-\beta t}\right)+M_{0}^{1-\sigma_{1}} \mathrm{e}^{-\beta t}\right]^{\sigma_{1} / 1-\sigma_{1}}\left[C_{1}-C M_{0}^{1-\sigma_{1}}\right] \mathrm{e}^{-\beta t^{\prime}}
\end{aligned}
$$

Hence at $t=0, \mathrm{~d} m / \mathrm{d} t=C_{1} M_{0} \sigma_{1}-C M_{0}$. This is actually the initial growth rate and can also be obtained by putting $m=M_{0}$ in Eq.11. We have $\mathrm{d} m / \mathrm{d} t \rightarrow 0$ as $t \rightarrow \infty$. Thus, the growth rate never becomes exactly zero although no growth is practically observed after a certain age. After reaching the peak value, $E_{g}$ decreases with time and, at a certain stage, it becomes too small to be practically measurable. From Eq.11, one can compute the mass (say $M_{g}$ ) for which $E_{g}$ has its highest value. This mass corresponds to the fastest growth rate and it is expressed as

$$
M_{g}=\left(C_{1} \sigma_{1} / C\right)^{1 / 1-\sigma_{1}} .
$$

Hence, the highest growth rate $\left(E_{g M}\right)$ is given by

$$
\begin{aligned}
E_{g M} & =E_{g}\left(\text { at } m=M_{g}\right) \\
& =C_{1}\left(C_{1} \sigma_{1} / C\right)^{\sigma_{1} / 1-\sigma_{1}}-C\left(C_{1} \sigma_{1} / C\right)^{1 / 1-\sigma_{1}} .
\end{aligned}
$$

Comparing $M_{g}$ with $M_{\text {opt }}$ in Eq.6A, we get $M_{g}<M_{\text {opt }}$, since $C_{3}>0$. It clearly implies that an organism attains the state of fastest growth before reaching the state of highest surplus power $\left(E_{s}\right)$.

Eq.13 expresses mass as a function of time. The surplus power $\left(E_{s}\right)$, being a function of mass, should also be a function of time. As $m$ approaches its saturation value $\left(M_{a}\right), E_{s}$ also approaches its saturation value $\left(\varepsilon_{s}\right)$. Substituting $m=M_{a}$ in Eq. 3 we get

$$
\varepsilon_{s}=C_{1}\left(C_{1} / C\right)^{\sigma_{1} / 1-\sigma_{1}}-C_{2}\left(C_{1} / C\right)^{1 / 1-\sigma_{1}} .
$$

Depending upon the growth parameters, there can be three different manners in which growth process can take place. These three possibilities are discussed below.

\section{CASE 1:}

The saturation mass $\left(M_{a}\right)$ can be smaller than the optimum mass $\left(M_{\text {opt }}\right)$. This case can be mathematically described as,

$$
M_{a}<M_{\text {opt }} \Rightarrow C_{3}>C_{2}\left[\left(1-\sigma_{1}\right) / \sigma_{1}\right] .
$$

The growth process, in this case, terminates before 
reaching the state of optimum mass $\left(M_{\text {opt }}\right)$. The saturation value $\left(\varepsilon_{s}\right)$ of the surplus power is smaller than $E_{s M}$.

\section{CASE 2:}

$$
M_{a}=M_{o p t} \Rightarrow C_{3}=C_{2}\left[\left(1-\sigma_{1}\right) / \sigma_{1}\right] .
$$

In this case, we have $\varepsilon_{s}=E_{s M}$ implying that after a sufficiently long time, the surplus power will almost remain at a constant level $E_{s M}$. As the growth process terminates the surplus power supply remains constant at its highest possible value. Therefore, the organism continues to live with the highest possible rate of surplus energy production.

\section{CASE 3:}

$$
M_{a}>M_{\text {opt }} \Rightarrow C_{3}<C_{2}\left[\left(1-\sigma_{1}\right) / \sigma_{1}\right] .
$$

Here the surplus energy saturates at a mass which exceeds the optimum mass. Then as like casel we again have $\varepsilon_{s}<E_{s M}$. Here, $E_{s}$ initially increases with time and after reaching the peak value $\left(E_{s M}\right)$ it decreases to its saturation value $\left(\varepsilon_{s}\right)$.

The conditions expressed by the Eqs.19-21 can be expressed by a single relation

$$
C_{3}=\rho C_{2}\left[\left(1-\sigma_{1}\right) / \sigma_{1}\right] \text {. }
$$

For the above three cases we have, $\rho>1, \rho=1$ and $0<\rho<1$ respectively.

Using Eqs.6A, 12 and 22 we define a quantity $R$ as

$$
R \equiv M_{a} / M_{o p t}=\left(1 /\left(\sigma_{1}+\rho\left(1-\sigma_{1}\right)\right)\right)^{1 / 1-\sigma_{1}}
$$

This ratio $(R)$ is a measure of the saturation mass relative to optimum mass. Since $\sigma_{1}<1$, we have $R \leq 1$ for $\rho \geq 1$ and $R>1$ for $\rho<1$. For $\rho \geq 1$, the energy allocation for growth process continues at most up to the optimum point. Beyond that point the surplus energy is allocated mainly for reproduction and other purposes. For $\rho<1$, the energy allocation for growth continues beyond the optimum point and there is a gradual shift in energy allocation from growth process to that for reproduction. Using Eqs.13 and 22 we get

$$
\begin{aligned}
& m(t) \\
& =\left[C_{1} \sigma_{1}\left(1-\mathrm{e}^{-\beta t}\right)\left(C_{2}\left(\sigma_{1}+\rho\left(1-\sigma_{1}\right)\right)\right)+M_{0}^{1-\sigma_{1}} \mathrm{e}^{-\beta t}\right]^{1 / 1-\sigma_{1}},
\end{aligned}
$$

where $\beta=C_{2}\left(1-\sigma_{1}\right)+\left(C_{2} \rho / \sigma_{1}\right)\left(1-\sigma_{1}\right)^{2}$.

Using the above expression of time-dependent mass, the surplus power $\left(E_{s}\right)$ can be expressed as,

$$
\begin{aligned}
& E_{s}=C_{1}\left[A\left(1-\mathrm{e}^{-\beta t}\right)+M_{0}^{1-\sigma_{1}} \mathrm{e}^{-\beta t}\right]^{\sigma_{1} / 1-\sigma_{1}} \\
& -C_{2}\left[A\left(1-\mathrm{e}^{-\beta t}\right)+M_{0}^{1-\sigma_{1}} \mathrm{e}^{-\beta t}\right]^{1 / 1-\sigma_{1}}
\end{aligned}
$$

where $A=C_{1} \sigma_{1} /\left(C_{2}\left(\sigma_{1}+\rho\left(1-\sigma_{1}\right)\right)\right)$ and $\beta=C_{2}\left(1-\sigma_{1}\right)+\left(C_{2} \rho / \sigma_{1}\right)\left(1-\sigma_{1}\right)^{2}$. Eq.25 shows how $E_{s}$, as a function of time, depends on the value of $\rho$.

Let us now define a time period $T_{\lambda}$ as the time required for the organism to attain a mass $m_{\lambda}$ where $m_{\lambda}=\lambda M_{a}$ and $0<\lambda<1$. Applying this definition and using Eq.14 one may obtain

$$
T_{\lambda}=1 /\left(C\left(1-\sigma_{1}\right)\right) \ln \left(\left(1-\left(M_{0} / M_{a}\right)^{1-\sigma_{1}}\right) /\left(1-\lambda^{1-\sigma_{1}}\right)\right) \text {. }
$$

In Eq.26, it is evident that as $\sigma_{1}$ increases, more time is required to attain a certain fraction $(\lambda)$ of the saturation mass $\left(M_{a}\right)$. For organisms with higher values of $\sigma_{1}$, the growth process continues for a longer time.

In the context of growth, one can define growth efficiency $\left(\varepsilon_{g}\right)$ as the ratio of the amount of surplus energy used for growth to the total surplus energy available at the moment, and can be expressed as,

$$
\begin{aligned}
& \varepsilon_{g}=E_{g} / E_{s}=1 / E_{s} \cdot \mathrm{d} m / \mathrm{d} t \\
& =\left(C_{1} m^{\sigma_{1}}-\left(C_{2}+C_{3}\right) m\right) / C_{1} m^{\sigma_{1}}-C_{2} m
\end{aligned}
$$

Since $\mathrm{d} m / \mathrm{d} t \rightarrow 0$ as $t \rightarrow \infty$, we must have $\varepsilon_{g} \rightarrow 0$ as $t \rightarrow \infty$.

In a similar fashion, one can define the reproduction efficiency $\left(\varepsilon_{p}\right)$ as the ratio of the amount of surplus energy used for reproduction to the total surplus energy available at the moment, and it can be expressed as

$$
\varepsilon_{p}=1-\varepsilon_{g}=C_{3} m /\left(C_{1} m^{\sigma_{1}}-C_{2} m\right) .
$$

As $t \rightarrow \infty, \varepsilon_{g} \rightarrow 0$ and therefore $\varepsilon_{p} \rightarrow 1$.

It is consistent with the practical observation that, as mass increases the proportion of energy allocation for growth decreases and the energy allocation for reproduction increases.

The rate of change of growth and reproduction efficiencies with respect to mass can be expressed as

$$
\mathrm{d} \varepsilon_{p} / \mathrm{d} m=-\mathrm{d} \varepsilon_{g} / \mathrm{d} m=C_{1} C_{3} m^{\sigma_{1}}\left(1-\sigma_{1}\right) /\left(C_{1} m^{\sigma_{1}}-C_{2} m\right)^{2}
$$

Since $\sigma_{1}<1$, the right hand side of Eq.29 is a positive quantity. Therefore, as mass increases, $\varepsilon_{p}$ continues to increase and $\varepsilon_{g}$ continues to decrease. Eq.29 suggests that, under no circumstances, $\mathrm{d} \varepsilon_{p} / \mathrm{d} m$ and $\mathrm{d} \varepsilon_{g} / \mathrm{d} m$ can be equal to zero. As a result one concludes that an organism, in its life span, never attains a mass for which its reproduction (or, growth) efficiency would be a maximum. In different organisms the growth efficiency seems to have a universal dependence on relative body mass [31,32]. Using the small amount of available data, Makarieva et al. [27] has concluded that there is a negative correlation between growth efficiency and metabolic 
rate. So the conclusion, drawn from Eq.28 is in good agreement with the literature in this topic.

The time taken by the organism to reach the state of highest growth rate can be determined by substituting $m=M_{g}$ in Eq.13 from Eq.16. This span of time, denoted by $T_{h}$ is

$$
\begin{aligned}
& T_{h}=\beta^{-1} \ln \left[\left(C_{1} \sigma_{1} / C-C_{1} / C\right)^{-1}\left(M_{0}^{1-\sigma_{1}}-C_{1} / C\right)\right] \\
& =\beta^{-1} \ln \left[\left(\sigma_{1}-1\right)^{-1}\left(M_{a}^{\sigma_{1}-1} M_{0}^{1-\sigma_{1}}-1\right)\right]
\end{aligned}
$$

\section{A Special Case: Length-Biomass Allometry}

In our recent study of length-biomass allometry of bidimensional seaweeds we have shown that the variation of length with time can be described properly in terms of two length parameters perpendicular to each other [33]. These are actually the sides of the smallest rectangle that can enclose the organism. This theoretical analysis was made in an attempt to explain the experimental findings of Scrosati on flat seaweeds [34]. According to this theoretical model, these two length parameters (say $L_{1}$ and $L_{2}$ ) has a power-law relation between them. This relation is given by,

$$
L_{2}=k\left(L_{1}\right)^{\lambda} .
$$

Here, $k$ is a constant of proportionality. From experimental observations, an average estimate of $\lambda$ was found to be 1.119 for the organisms described in our article [33]. These length parameters, $L_{1}$ and $L_{2}$, have separate allometric relations with the mass of the organism. In the present article we have explored the masstime relationship. Therefore, one can now formulate the length-time relationship of such species.

For the species of bi-dimensional seaweeds described in that article [33], an average estimate of the lengthbiomass allometry can be expressed by the following equations.

$$
\begin{aligned}
L_{1} & =7.811 \mathrm{~m}^{0.472} . \\
L_{2}=k\left(L_{1}\right)^{\lambda} & =9.976 \mathrm{~km}^{0.528} .
\end{aligned}
$$

The mass $(m)$ in the above equations is a function of time and its variation with time is described by the Eqs. 13 and $\mathbf{1 4}$ of the present study. It is a common observation that growth does not take place identically along two perpendicular directions in any flat organism. From the above equations the rates of growth along these directions can be expressed as

$$
\begin{aligned}
& \mathrm{d} L_{1} / \mathrm{d} t=3.687 m^{-0.528} \mathrm{~d} m / \mathrm{d} t, \\
& \mathrm{~d} L_{2} / \mathrm{d} t=5.267 \mathrm{~km}^{-0.472} \mathrm{~d} m / \mathrm{d} t .
\end{aligned}
$$

Here, $\mathrm{d} m / \mathrm{d} t$ is a function of time. Its dependence on time is expressed by Eq.15 of the present study.

\section{GRAPHICAL DEPICTION AND ANALYSIS}

Using the expressions derived in this article, we have illustrated various growth features graphically.

Figure 1 shows the general nature of dependence of mass $(m)$ and growth rate $(\mathrm{d} m / \mathrm{d} t)$ on time. These graphs are based on the Eqs.13 and 15. The mass initially increases rapidly with time and, after a sufficiently long time, it becomes asymptotic to the value of $M_{a}$. The rate of growth $(\mathrm{d} m / \mathrm{d} t)$ has a very sharp rise at the initial stage and, after reaching its peak value, it decreases slowly, becoming negligible after a sufficiently long time. As $t \rightarrow \infty$, we have $m \rightarrow M_{a}$ and $\mathrm{d} m / \mathrm{d} t \rightarrow 0$. This figure shows that the growth process never stops but it becomes so slow that it does not remain perceptible after a certain point of time (such as, at nearly $t=5$ in Figure 1).

Figure 2 shows the variation of growth rate as a function of mass for different values of the scaling exponent $\sigma_{1}$. For higher values of $\sigma_{1}$, the growth rate is higher and the duration of growth process is longer. At the very initial stage of growth, the rise in growth rate is almost independent of $\sigma_{1}$. The time required for attaining the peak rate is longer for higher values of $\sigma_{1}$. After reaching the peak value, the growth rate decreases but it does not fall as rapidly as it rises at the initial stage.

The graphs in Figure 3 show the change of mass of an organism with time for different values of the constant $C_{1}$. As time goes on, the mass $(m)$ becomes closer and

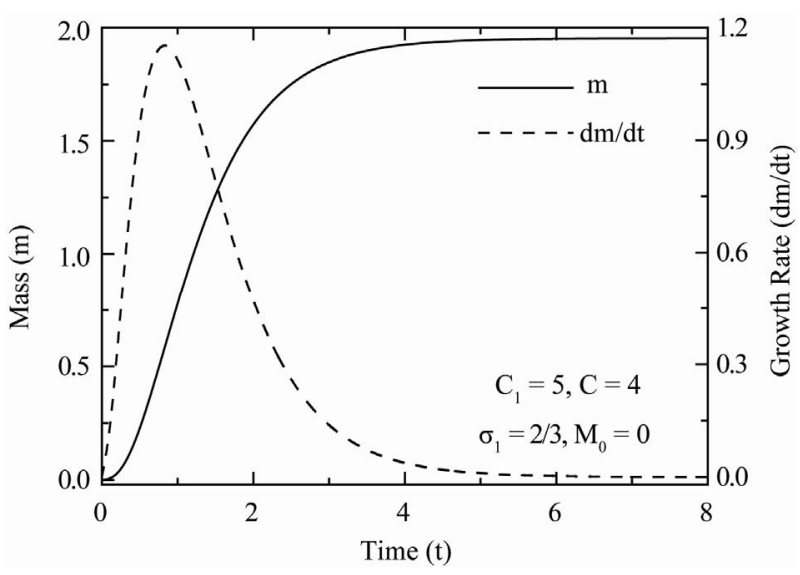

Figure 1. It shows the variation of mass and growth rate as functions of time. Here, the mass increases with a gradually decreasing rate and finally it becomes asymptotic to the value of $M_{a}$. The rate of growth rises fast at the initial stage; reaches its peak value and then continues to decrease slowly. At $t \rightarrow \infty$, we have $m \rightarrow M_{a}$ and $\mathrm{d} m / \mathrm{d} t \rightarrow 0$. In this case the value of $M_{a}$ is 1.953 . The growth rate reaches its highest value at $m=M_{g}=0.579$. 


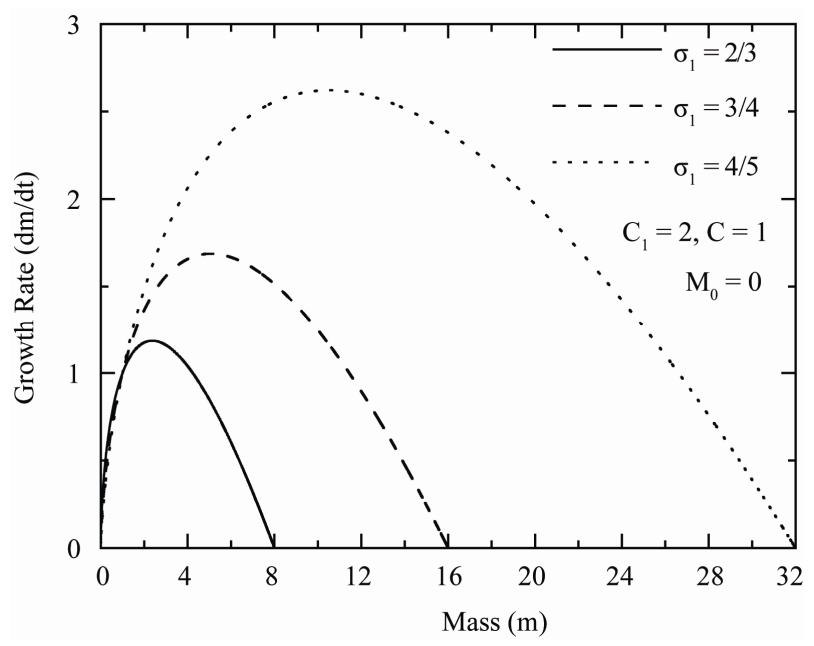

Figure 2. These plots show the change of the growth rate with mass for different values of the scaling exponent $\sigma_{1}$. For higher values of $\sigma_{1}$, the growth process continues for a longer time and the growth rate becomes higher. At the very initial stage of growth, the rise in growth rate seems to be almost independent of $\sigma_{1}$. As $\sigma_{1}$ increases, the time required for attaining the peak rate increases. After reaching the peak value, the growth rate decreases but it does not fall as rapidly as it rises at the initial stage. The values of $M_{g}$ for these three cases are 2.370, 5.063 and 10.486 .

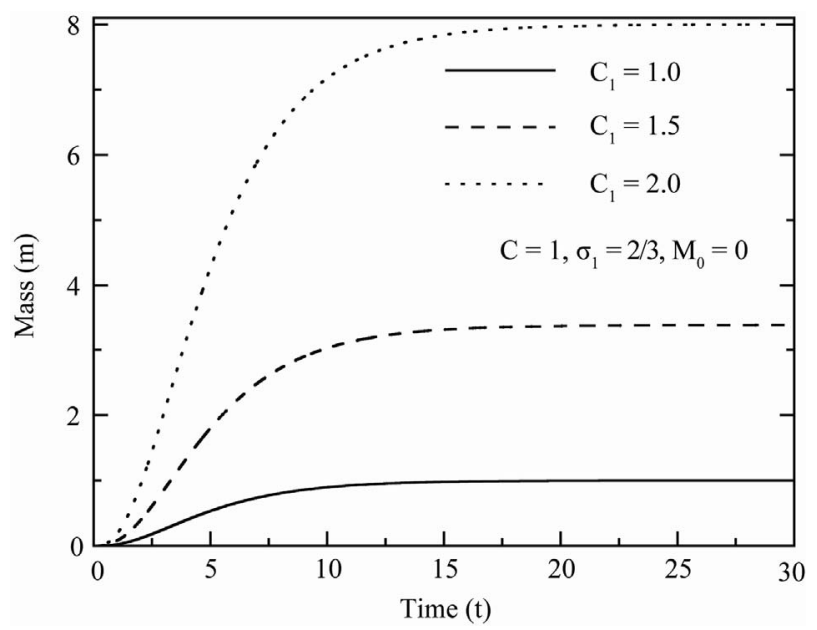

Figure 3. These plots show how the mass of an organism increases with time for different values of the constant $C_{1}$. The mass $(m)$ approaches a saturation level $\left(M_{a}\right)$ which increases with a rise in $C_{1}$. After a certain point of time the rise in mass becomes so slow that practically no growth can be observed. The values of $M_{a}$ for these three cases are 1, 3.375 and 8 .

closer to its saturation level $\left(M_{a}\right)$ which increases with a rise in $C_{1}$. After a long time the mass changes so slowly than no growth can be practically observed. The values of $M_{a}$ for these three cases are 1, 3.375 and 8 . These values are consistent with Eq.12, according to which, $M_{a}$ increase as the ratio $C_{1} / C$ and $\sigma_{1}$ become larger.

The graphs in Figure 4 show the dependence of growth rate on time for different values of the constant $C_{1}$. It is evident from these graphs that the time required for attaining the peak growth rate is independent of $C_{1}$. For higher values of this constant, the growth rate is higher at any stage of the growth process. The change in mass remains perceptible until $\mathrm{d} m / \mathrm{d} t$ becomes negligible. This effective termination point of growth is found to be the same for the cases shown in this figure and in Figure 3. Therefore, the effective duration of growth process is independent of $C_{1}$.

Figure 5 shows the dependence of growth rate on time for different values of the constant $C$. As $C$ increases, the growth rate becomes smaller and the effective duration of growth process becomes shorter. It is found in these graphs that, as $C$ increases, the time to reach the peak rate becomes shorter. According to Eqs.13 and 15, as $C$ increases, the value of $\beta$ increases and hence the organism approaches the effective termination point faster.

Figure 6 shows the variation of growth rate with time for different values of the scaling exponent $\sigma_{1}$. For higher values of $\sigma_{1}$, the effective duration of growth process is longer. At the very early stage of growth, an organism with smaller value of $\sigma_{1}$ has greater growth rate. Apart from this stage, organisms with higher values of $\sigma_{1}$ have larger growth rates in general. An organism with larger value of $\sigma_{1}$ takes more time in attaining the peak growth rate.

The variation of surplus power with time, for different values of the $\rho$, is shown in Figure 7. For $\rho=1, E_{s}$



Figure 4. This figure shows the variation of growth rate with time for different values of the constant $C_{1}$. For higher values of this constant, the growth rate becomes higher at any stage of the growth process. For these three cases, the growth rates attain their respective peaks at the same time. The increase in mass remains perceptible up to a certain point where $\mathrm{d} m / \mathrm{d} t$ is almost zero. This effective termination point of growth is found to be the same for the cases shown in this figure. Thus, the effective duration of growth process is independent of $C_{1}$. 


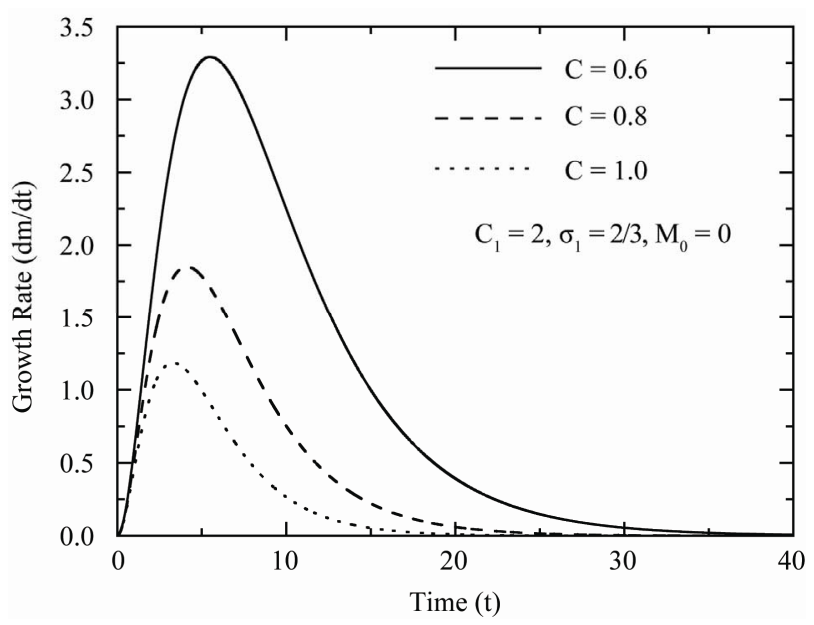

Figure 5. These plots show the variation of growth rate with time for different values of the constant $C$. For higher values of this constant, the growth process becomes slower at any stage of the growth process. It is evident from the graphs that as $C$ increases, the time required for attaining the peak rate becomes shorter. The increase in mass remains perceptible up to a certain point where $\mathrm{d} m / \mathrm{d} t$ is almost zero. For higher values of $C$, the organism approaches this effective termination point with greater rapidity. Thus, the effective duration of growth process becomes shorter for higher values of $C$.

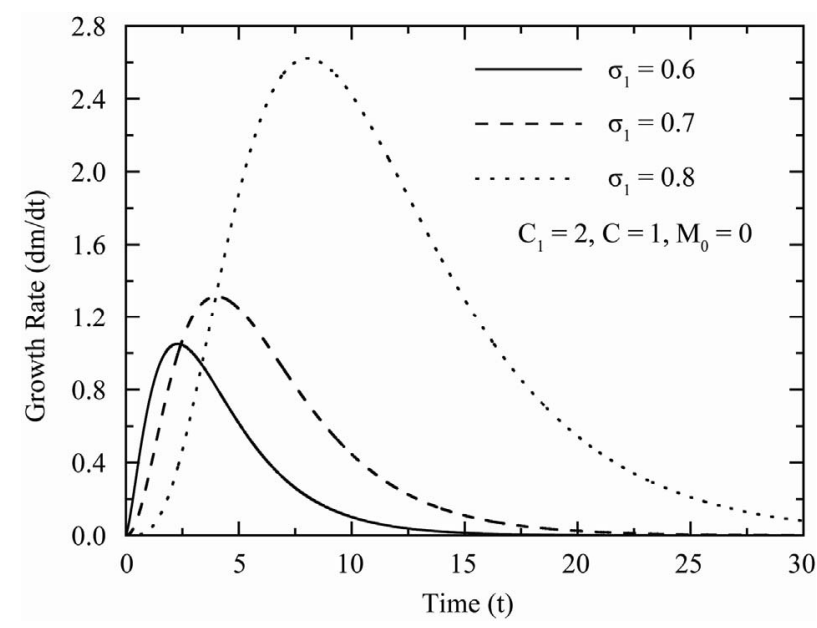

Figure 6. This figure shows the variation of growth rate with time for different values of the scaling exponent $\sigma_{1}$. It is clearly evident from these graphs that, as $\sigma_{1}$ increases, the effective duration of growth process increases. At the very early stage of growth, an organism with smaller value of $\sigma_{1}$ has greater growth rate. Except for this very small period, growth rate is higher for larger values of $\sigma_{1}$. For higher values of $\sigma_{1}$, the organism takes more time to attain the state of largest growth rate.

tends to reach the highest possible surplus power $\left(E_{s M}\right)$, implying that the organism continues to live with the largest supply of surplus power. For $\rho<1, E_{s}$ initially increases with time, reaches the peak value at $m=M_{\text {opt }}$ and, beyond that point, it attains saturation at a level

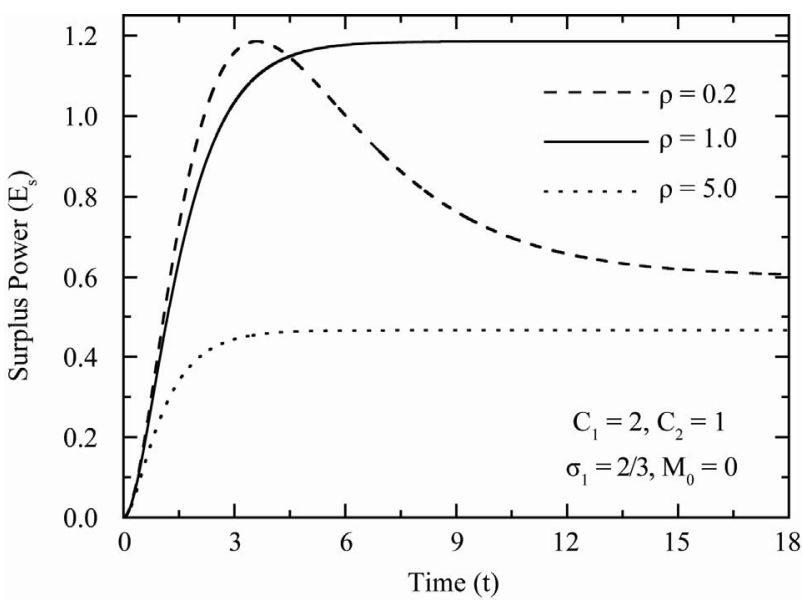

Figure 7. This figure shows the variation of surplus power with time for different values of the constant $\rho$. For $\rho=1$ we have $M_{a}=M_{o p t}$, for $\rho<1$ we have $M_{a}>M_{\text {opt }}$ and for $\rho>1$ we have $M_{a}<M_{\text {opt }}$. For $\rho=1$, the saturation value of $E_{s}$ is equal to the highest possible surplus power $\left(E_{s M}\right)$. For $\rho<1, E_{s}$ initially increases with time, reaching its peak value $\left(E_{s M}\right)$ at $m=M_{\text {opt }}$ and then decreases to saturate at a level smaller than $E_{s M}$. For $\rho$ $>1$, the mass saturates at a level smaller than $M_{o p t}$ and consequently, $E_{S}$ saturates at a level smaller than $E_{S M}$.

smaller than $E_{s M}$. For $\rho>1$, the mass attains its saturation level before reaching the value of $M_{o p t}$ and therefore, $E_{s}$ saturates at a level smaller than $E_{s M}$.

Figure 8 shows the change of growth efficiency $\left(\varepsilon_{g}\right)$ and reproduction efficiency $\left(\varepsilon_{p}\right)$ with respect to the ratio $m / M_{a}$, which is actually a measure of mass relative to its saturation value. As the growth process proceeds towards completion, this ratio $\left(m / M_{a}\right)$ approaches unity. This ratio, therefore, is a measure of the degree of completion of the growth process in an organism. As growth continues, $\varepsilon_{g}$ decreases from 1 to 0 and $\varepsilon_{p}$ increases from 0 to 1 . With a rise in mass, the utilization of surplus energy for reproduction increases and its allocation for growth decreases.

Figure 9 show the variation of growth efficiency with time for different values of the constant $\rho$. At $t=0$, the efficiency has the highest value (i.e. unity), for any value of $\rho$. The growth efficiency decreases with time and it approaches its lowest value (i.e. zero) as $t \rightarrow \infty$, for any value of $\rho$. As $\rho$ increases, the growth efficiency decreases faster with time. For higher values of $\rho$, the growth efficiency is smaller at any stage of the growth process. Thus, for larger values of $\rho$, the utilization of surplus energy for growth is smaller.

Figure 10 shows the variation of $T_{\lambda}$ as a function of $\lambda$, for different values of the scaling exponent $\sigma_{1}$. For any value of $\lambda, T_{\lambda}$ is larger for higher values of $\sigma_{1}$. Near the right edge of the above frame, a vertical line has been drawn to mark the point where the mass attains $95 \%$ of its saturation level $\left(M_{a}\right)$. Near this point, the 


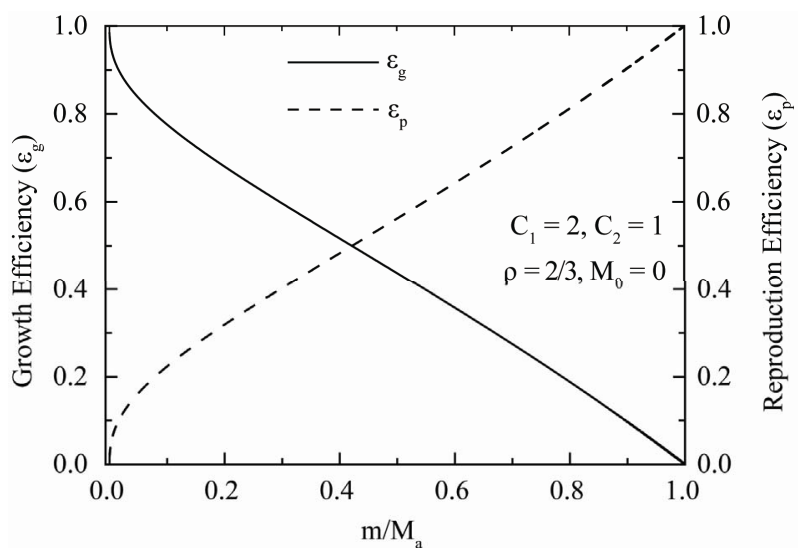

Figure 8. This figure shows the variations of growth efficiency $\left(\varepsilon_{g}\right)$ and reproduction efficiency $\left(\varepsilon_{p}\right)$ with respect to the ratio $m / M_{a}$. As the growth process approaches termination, this ratio $\left(m / M_{a}\right)$ approaches unity. As growth continues, $\varepsilon_{g}$ decreases from 1 to 0 and $\varepsilon_{p}$ increases from 0 to 1 . With a rise in mass, the utilization of surplus energy for reproduction increases and its allocation for growth decreases.

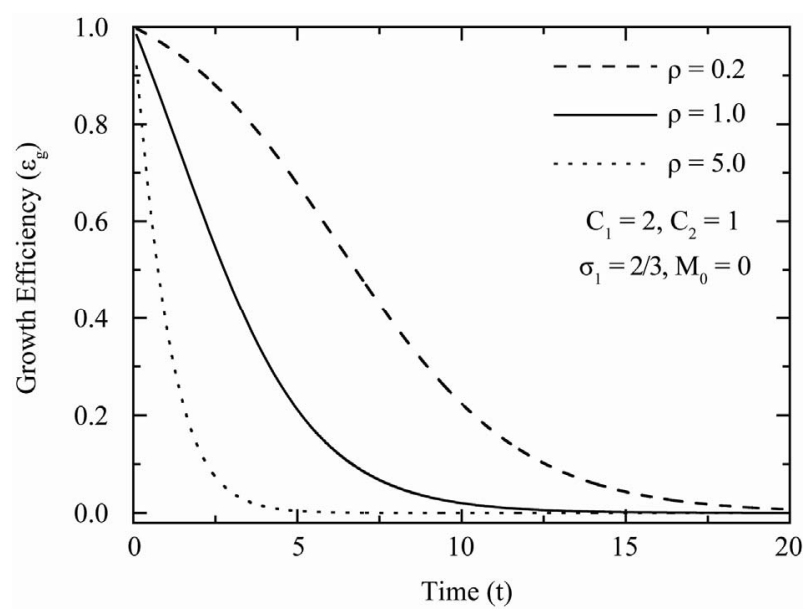

Figure 9. These graphs show the variation of growth efficiency with time for different values of the constant $\rho$. At $t=0$, the efficiency has the highest value (i.e. unity), irrespective of the value of $\rho$. The growth efficiency is found to decrease with time and it approaches its lowest value (i.e. zero) as $t \rightarrow \infty$ in all these three cases. As $\rho$ becomes larger, the growth efficiency falls more rapidly with time. For higher values of $\rho$, the growth efficiency is smaller at any stage of the growth process. Thus, for larger values of $\rho$, smaller fraction of surplus energy is utilized of for growth.

slopes of these curves become extremely high, implying the fact that a very long time is required for a slight change in mass. This vertical line almost marks the effective termination point of growth because any practical observation (or measurement) of growth becomes more and more difficult at this stage.

Figure 11 shows the variation of the length parameters $\left(L_{1}\right.$ and $\left.L_{2}\right)$ of a bi-dimensional organism with time. These plots are based on the Eqs.32A and B for $L_{1}$

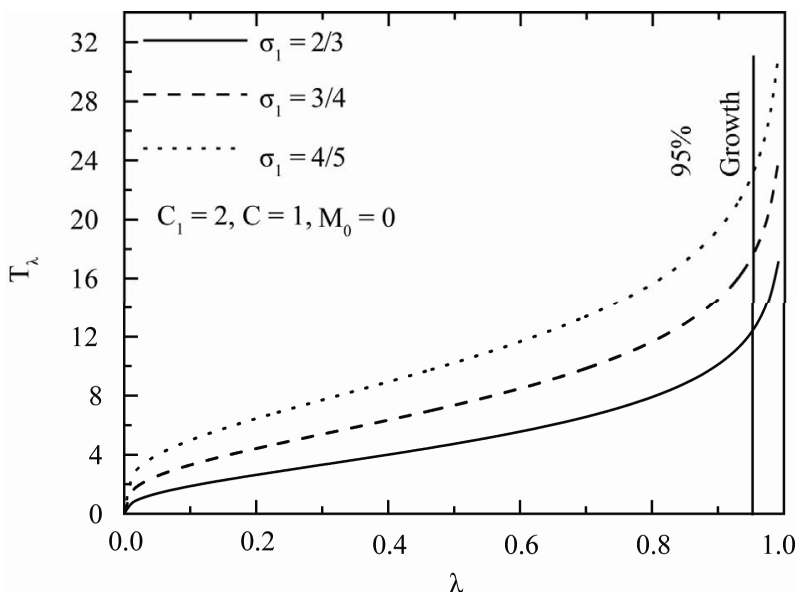

Figure 10. This figure shows the variation of $T_{\lambda}$ as a function of $\lambda$, for different values of the scaling exponent $\sigma_{1}$. For any value of $\lambda, T_{\lambda}$ is larger for higher values of $\sigma_{1}$. The vertical line, near the right edge of the frame, mark the point where $m=0.95$ $\mathrm{M}_{\mathrm{a}}$. Very high slope near this point implies that practically very little rise in mass is observable at this stage of the growth process.

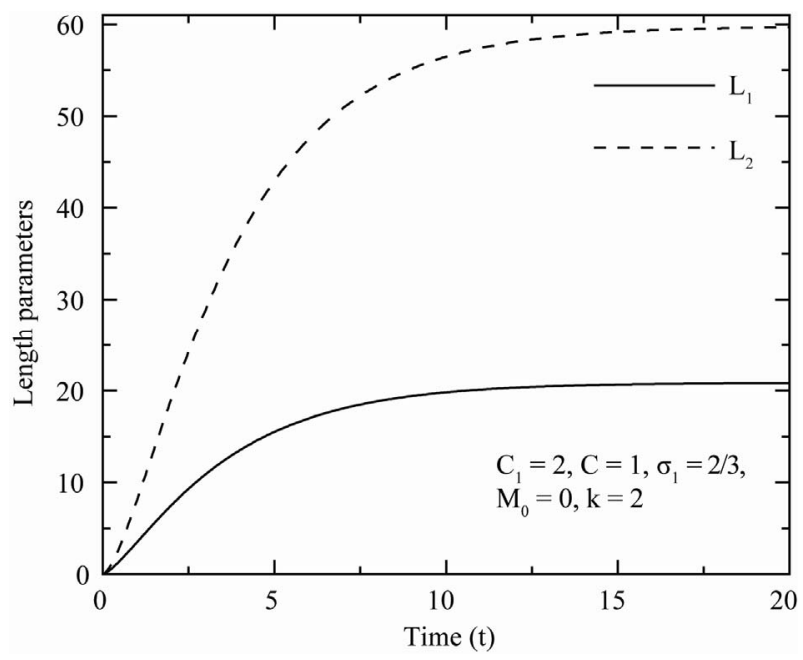

Figure 11. This figure shows the variation of the length parameters $\left(L_{1}\right.$ and $\left.L_{2}\right)$ of bi-dimensional organisms with time. One of the parameters attains the state of saturation earlier than the other.

and $L_{2}$ respectively. Here, the time dependence of mass $(m)$ has been obtained from Eq.13. One of the parameters attains the state of saturation earlier than the other, which is quite consistent with our observations.

Figure 12 shows the rate of change of two length parameters of a bi-dimensional organism as functions of time. These plots are based on the Eqs.33A and $\mathbf{B}$ for $L_{1}$ and $L_{2}$ respectively. Here, the time dependence of the growth rate $(\mathrm{d} m / \mathrm{d} t)$ has been obtained from Eq.15. Any of these rates attains a maximum value and then decreases to zero asymptotically. One of the rates attains peak value earlier and goes to zero faster than the other 


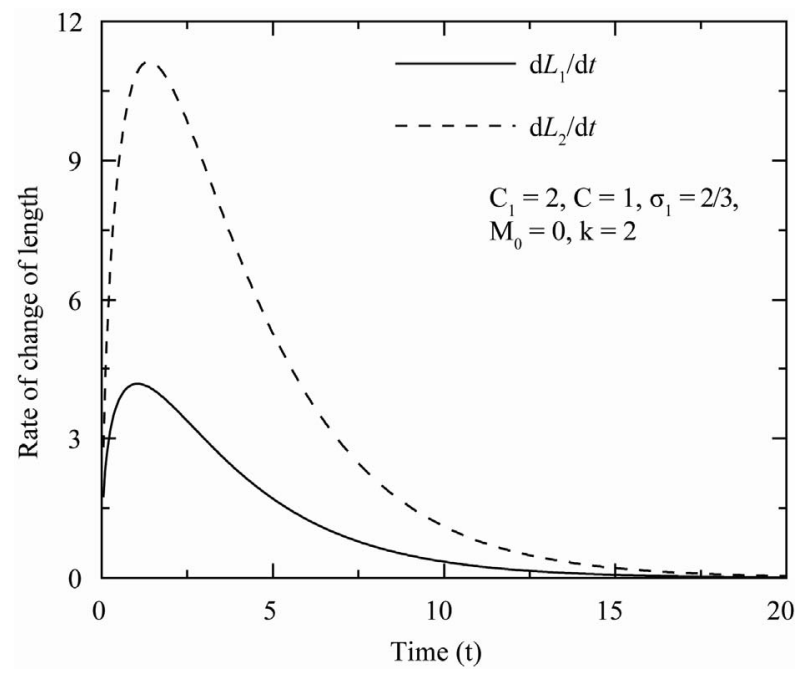

Figure 12. This figure shows the rate of change of two length parameters of bi-dimensional organisms as functions of time. The rate attains a maximum value and then decreases to zero asymptotically. One of the rates attains peak value earlier and goes to zero faster than the other parameter.

parameter.

An important fact, regarding the constant $C_{3}$, comes out in this growth model. Using Eqs.6A and $\mathbf{1 1}$ it is found that, at $m=M_{\text {opt }}$ this constant is given by

$$
C_{3}=-\mathrm{d} E_{g} / \mathrm{d} m=-\mathrm{d} / \mathrm{d} m(\mathrm{~d} m / \mathrm{d} t) .
$$

Using Eq.34, one can determine the value of $C_{3}$ experimentally.

\section{CONCLUSIONS}

An organism must always have some surplus energy $\left(E_{s}\right)$ for a healthy survival which involves processes like repair, maintenance and coping with environmental fluctuations etc. apart from growth and reproduction phenomena. Therefore, the net amount of surplus energy or the mass specific surplus energy may be the determining factors for mortality of an organism. For such quantities, there should be specified limit below which the survival of the organism is not possible and the limiting value may be different for different species. Generally, a part of surplus energy is always converted into mass, causing an enhancement in size of the organism. There should be an extensive experimental investigation to find out the functional dependence of reproduction (or, growth) efficiency on body mass. Through the present mathematical formulations, we have shown that biological growth process can take place in three possible modes, depending on the relationships among various parameters. Using this model, we have analyzed the mechanism of variation of length with age of a bi-dimensional organism where growth takes place essentially along two dimensions. The usefulness of this model is that, using the expressions of $M_{a}, M_{g}, T_{\lambda}, T_{h}$ it would be possible to determine the values of $\sigma_{1}, C_{1}, C_{2}$ and $C_{3}$ from experimental observations, leading to a deeper insight into the energy allocation for different physiological purposes.

\section{REFERENCES}

[1] Hedin, L.O. (2006) Physiology: Plants on a different scale. Nature, 439, 399-400. doi:10.1038/439399a

[2] Harris, L.A., Duarte, C.M. and Nixon, S.W. (2006) Allometric laws and prediction in estuarine and coastal ecology. Estuaries and Coasts, 29, 340-344. doi:10.1007/BF02782002

[3] McKechnie, A.E., Freckleton, R.P. and Jetz, W. (2006) Phenotypic plasticity in the scaling of avian basal metabolic rate. Proceedings of the Royal Society B, 273, 931-937. doi:10.1098/rspb.2005.3415

[4] Van der Meer, J. (2006) Metabolic theories in ecology. Trends in Ecology and Evolution, 21, 136-140. doi:10.1016/j.tree.2005.11.004

[5] West, G.B., Woodruff, W.H. and Brown, J.H. (2002) Allometric scaling of metabolic rate from molecules and mitochondria to cells and mammals. Proceedings of the National Academy of Sciences of the USA, 99, 2473-2478. doi:10.1073/pnas.012579799

[6] Kozlowski, J. (1992) Optimal allocation of resources to growth and reproduction: Implications for age and size at maturity. Trends in Ecology \& Evolution, 7, 15-19. doi:10.1016/0169-5347(92)90192-E

[7] Sebens, K.P. (2002) Energetic constraints, size gradients, and size limits in benthic marine invertebrates. Integrative and Comparative Biology, 42, 853-861. doi:10.1093/icb/42.4.853

[8] Kozlowski, J. and Weiner, J. (1997) Interspecific allometries are by-products of body size optimization. American Naturalist, 149, 352-380. doi:10.1086/285994

[9] Castorina, P., Delsanto, P.P. and Guiot, C. (2006) Classification scheme for phenomenological universalities in growth problems in physics and other sciences. Physical Review Letters, 96, 188701-188704. doi:10.1103/PhysRevLett.96.188701

[10] Gompertz, B. (1825) On the nature of the function expressive of the law of human mortality and on the mode of determining the value of life contingencies. Philosophical Transactions of the Royal Society, 115, 513-585. doi:10.1098/rstl.1825.0026

[11] Steel, G.G. (1977) Growth kinetics of tumors. Clarendon Press, Oxford.

[12] Weldom, T.E. (1988) Mathematical model in cancer rersearch. Adam Hilger Publisher, Briston.

[13] Von Bertalanffy, L. (1960) Principles and theory of growth. In: Nowinski, W.W., Ed., Fundamental Aspects of Normal and Malignant Growth, Elsevier, Amsterdam, 137-259.

[14] Von Bertalanffy, L. (1938) A quantitative theory of organic growth (inquiries on growth laws II). Human Biology, 10, 181- 213.

[15] Brown, J.H., Gillooly, J.F., Allen, A.P., Savage, V.M. and West, G.B. (2004) Toward a metabolic theory of ecology. Ecology, 85, 1771-1789. doi:10.1890/03-9000 
[16] Kooijman, S.A.L.M. (2010) Dynamic energy budget theory for metabolic organisation. 3rd Edition, Cambridge University Press, Cambridge.

[17] Sousa, T., Domingos, T. and Kooijman, S.A.L.M. (2008) From empirical patterns to theory: A formal metabolic theory of life. Philosophical Transactions of the Royal Society B, 363, 2453-2464. doi:10.1098/rstb.2007.2230

[18] Rau, A.R.P. (2002) Biological scaling and physics. Journal of Biosciences, 27, 475-478. doi:10.1007/BF02705043

[19] Demetrius, L. (2006) The origin of allometric scaling laws in biology. Journal of Theoretical Biology, 243, 455-467. doi:10.1016/j.jtbi.2006.05.031

[20] Vogel, S. (2004) Living in a physical world. Journal of Biosciences, 29, 391-397. doi:10.1007/BF02712110

[21] Da Silva, J.K.L. and Barbosa, L.A. (2007) Non-universal Interspecific Allometric Scaling of Metabolism. Journal of Physics A, 40, F1-F7.

[22] Da Silva, J.K.L. and Barbosa, L.A. (2009) Non-universal interspecific allometric scaling of metabolism. Brazilian Journal of Physics, 39, 699-706.

[23] Economos, A.C. (1982) On the origin of biological similarity. Journal of Theoretical Biology, 94, 25-60. doi:10.1016/0022-5193(82)90328-9

[24] Biswas, D., Das, S.K. and Roy, S. (2008) Importance of scaling exponents and other parameters in growth mechanism: An analytical approach. Theory in Biosciences, 127, 271-276. doi:10.1007/s12064-008-0045-9

[25] Biswas, D., Das, S.K. and Roy, S. (200/8) Dependence of the individual growth process upon allometric scaling exponents and other parameters. Journal of Biological Systems, 16, 151-163. doi:10.1142/S0218339008002411

[26] West, G.B., Brown, J.H. and Enquist, B.J. (2004) Growth model based on first principle or phenomenology. Functional Ecology, 18, 188-196. doi:10.1111/j.0269-8463.2004.00857.x

[27] Makarieva, A.M., Gorshkov, V.G. and Li, B.-L. (2004) Ontogenetic growth: Models and theory. Ecological Modelling, 176, 15-26. doi:10.1016/i.ecolmodel.2003.09.037

[28] Warren, C.E. and Davis, G.E. (1967) Laboratory studies on the feeding bioenergetics and growth of fish. In: Gerking, S.D., Ed., The Biological Basis for Freshwater Fish Production, Blackwell, Oxford, 175-214.

[29] Sebens, K.P. (1979) The energetics of asexual reproduction and colony formation in benthic marine invertebrates. Integrative and Comparative Biology, 19, 683-697. doi:10.1093/icb/19.3.683

[30] Sebens, K.P. (1982) The limits to indeterminate growth: An optimal size model applied to passive suspension feeders. Ecology, 63, 209-222. doi:10.2307/1937045

[31] Pandian, T.J. and Vernberg, F.J. (1987) Animal energetics. Academic Press, San Diego.

[32] Brody, S. (1945) Biogenetics and growth. Reinhold Publishing Corporation, New York.

[33] Majumdar, P. and Roy, S. (2010) A theoretical interpretation of length-biomass allometry of predominantly bidimensional seaweeds. Journal of Theoretical Biology, 266, 226-230. doi:10.1016/j.jtbi.2010.06.035

[34] Scrosati, R. (2006) Length-biomass allometry in primary producers: Predominantly bidimensional seaweeds differ from the "universal" interspecific trend defined by microalgae and vascular plants. Canadian Journal of Botany, 84, 1159-1166. doi:10.1139/b06-077 